\title{
Review of applications and challenges of quantitative systems pharmacology modeling and machine learning for heart failure
}

\author{
Limei Cheng $^{1}$ (D) Yuchi Qiu $^{2} \cdot$ Brian J. Schmidt $^{1} \cdot$ Guo-Wei Wei $^{2,3,4}$
}

Received: 24 June 2021 / Accepted: 22 September 2021 / Published online: 12 October 2021

(c) The Author(s) 2021

\begin{abstract}
Quantitative systems pharmacology (QSP) is an important approach in pharmaceutical research and development that facilitates in silico generation of quantitative mechanistic hypotheses and enables in silico trials. As demonstrated by applications from numerous industry groups and interest from regulatory authorities, QSP is becoming an increasingly critical component in clinical drug development. With rapidly evolving computational tools and methods, QSP modeling has achieved important progress in pharmaceutical research and development, including for heart failure (HF). However, various challenges exist in the QSP modeling and clinical characterization of HF. Machine/deep learning (ML/DL) methods have had success in a wide variety of fields and disciplines. They provide data-driven approaches in HF diagnosis and modeling, and offer a novel strategy to inform QSP model development and calibration. The combination of ML/DL and QSP modeling becomes an emergent direction in the understanding of HF and clinical development new therapies. In this work, we review the current status and achievement in QSP and ML/DL for HF, and discuss remaining challenges and future perspectives in the field.
\end{abstract}

Keywords Quantitative systems pharmacology $\cdot$ QSP modeling · Machine learning · Heart failure · Physiological modeling $\cdot$ Modeling and simulation

\section{Introduction}

\section{Heart failure}

Heart failure (HF) is generally described as a condition where the blood output of the heart is not sufficient to meet metabolic demands of the tissues [1]. However, a variety of dysfunctions comprise heart failure, heart failure can be a challenging syndrome to diagnose clinically, and

Limei Cheng

limei.cheng@bms.com

1 Quantitative Systems Pharmacology and Physiologically Based Pharmacokinetics, Bristol Myers Squibb, Princeton, NJ 08536, USA

2 Department of Mathematics, Michigan State University, East Lansing, MI 48824, USA

3 Department of Electrical and Computer Engineering, Michigan State University, East Lansing, MI 48824, USA

4 Department of Biochemistry and Molecular Biology, Michigan State University, East Lansing, MI 48824, USA compensatory cardiac mechanisms can sometimes mask an underlying disease state. A critical functional quantity used in the characterization of heart failure is the ejection fraction (EF), the ratio between the blood volume ejected and total filled volume. EF is usually reported for the cardiac chamber responsible for systemic delivery of arterial blood through the aorta, the left ventricle ejection fraction (LVEF). The two most commonly diagnosed types of heart failure are heart failure with reduced ejection fraction (HFrEF) and heart failure with preserved ejection fraction (HFpEF). Normally, the EF of a healthy person is $55-70 \%$, and when EF drops below $40 \%$, it is characterized as HFrEF. Intermediate ejection fractions of $40-55 \%$ are often considered to be abnormal [2]. For $\mathrm{HFpEF}$ patients both the blood ejected volume and the total filled blood volume are reduced, which results in a near-normal, preserved EF.

Heart failure can have a number of etiologies, but generally progresses from an initial cardiac injury through a remodeling stage that ultimately results in cardiac dysfunction [3]. The causes of cardiac injury can include 
myocardial disease, pericardial or endocardial abnormalities, valve diseases such as regurgitation and stenosis, arrhythmia, pressure overload, or volume overload [4]. The most common cause is cardiac injury is myocardial ischemia, which usually involves blockage of the coronary arteries from coronary artery disease (CAD) [4]. Remodeling is driven by cellular processes including cardiomyocyte hypertrophy, proliferation of fibroblasts, extracellular matrix changes including an initiation of fibrosis, apoptosis and necrosis of myocytes, and inflammation [4]. Hypertrophy can be stimulated by reductions in blood flow to the heart, which results in increase oxygen demand. In addition to increased demand for oxygen with additional muscle tissue, the increased wall stiffness can serve as a barrier to sufficient coronary blood flow. This positive cycle causes cardiac remodeling and is often associated with worsening in HFpEF. Cardiac myocyte apoptosis and necrosis results in weakened heart muscle, reduced force of contraction, reduced stroke volume, and ultimately produces remodeling with progressively thinner walls and larger ventricle chambers in HFrEF [5].

Increases in fluid retention and congestion are additional hallmarks of heart failure. When the heart does not contract properly in heart failure, less blood passes through the heart and lungs. The kidney compensates for the response in arterial underfilling as triggered by baroreceptor activation as well as neurohormonal stimulation, increasing sodium and water retention. Gradually, the interstitial fluid and plasma volume expand [6]. This results in congestion in the lungs and body and fluid retention in tissues and organs. In addition to the loss of cardiac functionality, heart failure therefore also progressively results in shortness of breath, swelling, and the loss of the ability to exercise.

No single mechanism can account for the heterogenous clinical syndromes and conditions of heart failure. Thus, multiple strategies are needed to assess the mechanisms, complex systems, describe the clinical syndromes, and assess the likelihood of the many alternate causes of heart failure [7].

\section{Introduction of quantitative systems pharmacology}

Mathematical biology and pharmacology models are increasingly used in preclinical and clinical drug development. Quantitative systems pharmacology (QSP) is an emerging interdisciplinary field that integrates systems biology and pharmacometrics, with an emphasis on dynamic modeling, to quantitatively predict the effects of clinical interventions and their combinations under a variety of genetic, biochemical, biophysical, biomechanical, and physiological conditions. Sometimes these mechanistic underpinnings are also mapped to influential demographic considerations such as gender, race, and age [8, 9]. Recently, machine learning (ML) approaches to inform QSP have been of increasing interest and utility. QSP models are developed by incorporating underlying disease and therapeutic mechanisms, and are leveraged to solve complex problems, improve decisions, and reduce costs in drug development. They are applied from early drug design and discovery to late-stage clinical development. A fast-growing interest in application of QSP to dosing and trial decision-making for clinical development has made QSP a well-recognized tool in drug development. QSP has supported clinical development in many therapeutic areas and provided unexpected insights [10]. The further integration, calibration, and validation with patient data, particularly those from clinical trials, enables QSP to provide mechanical and actionable predictions. QSP modeling has become an indispensable tool that has also created interest from regulatory agencies such as the US Food and Drug Administration (FDA) and European Medicines Agency (EMA) for new drug development $[10,11]$. As such, QSP will have a substantial impact on human healthcare and the pharmaceutical industry in the future.

\section{Overview of machine learning and deep learning}

Machine learning (ML), including deep learning, has had a growing and substantial impact on science, engineering, technology, and industry in the past decade $[12,13]$. It is a branch of artificial intelligence and computer science, which focuses on the use of data and algorithms to imitate the way that humans learn, gradually improving its accuracy [14]. Supervised learning and unsupervised learning are two major categories that have a wide clinical application on HF [15], although semi-supervised learning and self-supervised learning are potentially useful. In this section, we give a brief introduction to these areas of machine learning algorithms.

\section{Supervised learning}

The primary difference between supervised learning and unsupervised learning is whether the training data is "labeled." Supervised learning uses labeled datasets to train the ML model. Supervised learning consists of two major types of tasks, regression and classification, where a regression task predicts a continuous quantity and a classification task generally predicts a category. Linear regression is one basic regression algorithm that uses a linear approach to model the relationship between a scalar response and one or more explanatory variables (i.e. predictors) [16]. Logistic regression is used to model the probability of a certain class or event existing, which is 
mainly intended for classification problems [17]. Support vector machine (SVM) is a classification method that develops an optimal hyperplane that maximally separates high dimensional data points classified into two categories. Although the hyperplane is a linear divider, nonlinear classification problems can be tackled with a kernel function. New examples are then mapped into that same space and predicted to belong to a category based on which side of the line they fall [18]. SVM has also been adapted for regression problems, support vector regression (SVR). Another classification method is the decision tree, a flowchart-like structure with many binary decision points, or nodes. Leaf nodes are the end points in a decision tree structure, and each leaf node assigns a class label. The paths from the root to the leaf represent classification rules [19]. Decision trees have been noted to be prone to overfitting, whereby they may fit the data very well, including spurious features and noise. An ensemble method, i.e. the random forest method, proceeds by constructing multiple decision trees at training time and averaging predictions of the individual trees to mitigate overfitting issues [20]. Many additional ensemble learning methods have been developed, such as gradient boosting trees and XGBoost [21].

Deep learning has become more popular in supervised learning because of its capabilities for universal approximation and availability of increasingly powerful high-performing computational tools. One popular deep learning algorithm is the artificial neural network (ANN), a network consisting of connected units or nodes [22]. Each node produces a single output value based on a weighted sum of the inputs plus a bias term. Intermediate nodes between the input and output nodes are often called hidden nodes, and there may be multiple layers of intervening nodes. To prevent overfitting and reduce the dimensionality of the model in the neural network, convolution neural networks (CNN) introduced convolutional layers, which perform additional matrix multiplication operations on the input. CNNs are particularly designed for learning image data [23]. Recurrent neural networks (RNN) include additional recurrence relationships that rely on previous network time points that are not included in ANNs but are needed to help predict sequences, such as letters in sentences and words. In natural language processing, many RNN architectures were introduced to make the model scalable to sequence data with various lengths, such as long short-term memory (LSTM) [24], gated recurrent unit (GRU) [25] and transformer [26]. ML models can simultaneously take multiple features or biomarkers as input data, and meanwhile predict multiple clinical events or outputs. The existence of multiple output data enables the use of the so-called to multitask deep learning (MDL) method. This approach, also called transfer learning, can take the advantage of a large data set to help improve the prediction accuracy of small data sets.

\section{Unsupervised learning}

Another type of machine learning task is unsupervised learning, where the data have no labels. Two of the main methods in unsupervised learning are dimension reduction and clustering. Dimension reduction transforms high-dimensional data into a low-dimensional space retaining the most meaningful properties. Clustering divides data into multiple groups with similar internal characteristics. Dimension reduction methods are common in fields that deal with high-dimensional data for noise reduction, data visualization, and cluster analysis [27]. Linear dimension reduction, such as principal component analysis (PCA), performs a linear mapping of the data to eigenspace in lower dimension, maximally preserving the variability in the original data while using minimal dimensions in the transformed space [28]. Other nonlinear dimension reduction methods, such as non-negative matrix factorization (NMF) [29], T-distributed stochastic neighbor embedding (t-SNE) [30], autoencoder [31], and uniform manifold approximation and projection (UMAP) [32], were widely used to data with different structures [33]. Clustering is the task of grouping a set of objects in such a way that objects in the same group are more similar to each other than to those in other groups. Distance-based clustering [34, 35], hierarchical clustering [36], community-based clustering [37, 38], density-based clustering [39], soft clustering [40, 41], and graph-based clustering [42] were widely applied to transcriptomic data analysis [43], pattern recognition [44], image processing [45] as well as heart failure [46] to reveal data internal characteristics.

\section{QSP modeling for cardiovascular diseases and heart failure}

\section{Applications of QSP modeling for cardiovascular diseases and heart failure}

For pharmaceutical discovery and development, one crucial question that must be addressed is whether the potential treatment is safe and efficacious. Mechanistic systems modeling approaches can provide insights and guide target identification and drug evaluation throughout the drug development process [47]. QSP models can improve understanding of heart failure mechanisms, provide safety and efficacy assessments, and assist in the design and planning of clinical trials.

QSP modeling has been applied in the pharmaceutical industry to cardiovascular applications. As one example, 
the Cardiovascular Physiolab ${ }^{\circledR}$ platform models the biology and pathophysiology of cardiovascular disease and was applied to explore the progression of atherosclerosis and response to therapies [48]. One striking prediction from the model was that although cholesterol ester transfer protein (CETP) inhibition can modulate the circulating lipoprotein profile, the known mechanisms for clinically explored CETP inhibitors generally would not effectively remove cholesterol from plaque to facilitate improved cardiovascular outcomes. That is, the model both provided a quantitative hypothesis for the lack of impact of torcetrapib on plaque outcomes and further suggested a class effect posing a challenge to the clinical development of CETP inhibitors that would extend beyond torcetrapib. The mechanistic insight and predictions were made public prior to the readouts of multiple phase 3 trials for CETP inhibitors with outcomes in agreement with the insights provided by the mechanistic modeling work [47-49].

For heart failure patients, reduced cardiac output and arterial filling pressure leads to congestion in the lungs and body, which causes short breath and fluid retention, contributing to further volume overload. As one example of mechanistic modeling applied to heart failure, cardiac and renal interactions in HFrEF were modeled by $\mathrm{Yu}$ et al. to incorporate the underlying mechanisms of edema and investigate the effects of renally-targeted therapies on HFrEF [50]. The model investigated the poorly understood effects of sodium-glucose co-transporter 2 inhibitors (SGLT2i) on HF. Simulated virtual diabetic and non-diabetic HF patients and simulated clinical trials were used to evaluate the effect of SGLT2i on cardiac functions, blood volume, congestion, and edema in HFrEF patients. Yu et al. proposed that HFrEF improves with SGLT2 inhibition due by reducing cardiac preload and relieves congestion by reducing the interstitial fluid accumulation.

Myocardial energetics also change with decompensated heart failure. However, it is unknown how mechanical function and changes in myocardial energetics interact with each other in HF. Tewari et al. developed an integrated model with mitochondrial ATP energetics, calcium-dependent actin-myosin cross-bridge cycling, and systemic resistance to address this question [51]. The model was applied to investigate the efficacy of drugs, including omecamtive mecarbil, on cross-bridge cycling kinetics and resulting oxygen demands in decompensated heart failure. Tewari et al. used the computational analysis to propose how metabolic changes can account for the systolic dysfunction in heart failure.

QSP modeling in HF often uses data from literature, nonclinical studies, and available prior clinical data to inform model develop and assist clinical studies and development. There are many open questions about the development of therapeutics for heart failure that would benefit from a QSP modeling approach. QSP can address early assessments of clinical efficacy, assessments of the optimal dose level, dosing regimen optimization, combination with other therapeutics including the standard of care, and identification of characteristics of responders in heterogenous patient populations. QSP models can also be updated with emerging clinical data. When the primary outcome fails in a clinical trial, QSP can be applied to reassess whether there is a strong biologic rationale that favors the treatment and whether the failure was due to the trial population [52].

A summary diagram of a QSP model being applied to inform clinical development is shown in Fig. 1. The model mathematically describes the physiology of cardiovascular, respiratory, renal system, $\mathrm{Na}+$ /water regulation, and the central nervous and hormonal regulation system. The model can simulate a pulsatile heart and hemodynamics with interactions of neuro-hormonal control and kidney function of a virtual patient as a normal subject or with heart failure. In addition to questions about clinical dosing strategies, the heart failure model has been applied to generate a deeper biological and clinical understanding of the disease states in heart failure. The model has also been used to investigate the mechanisms of action for therapeutic targets in clinical development. The heart failure QSP modeling approaches integrate clinical and non-clinical data and current knowledge in a quantitative and mechanistic fashion to generate actionable predictions.

QSP models can simulate virtual patients (VPs) and one major application of the heart failure QSP modeling is also HF VP simulation. A variety of criteria are used to establish that simulated VPs are biologically plausible, or valid. Biomarkers of plausible VPs should be clinically observed physiological ranges, for example observed ranges of cardiac ejection fraction, left ventricle volume, total peripheral resistance, heart rate, cardiac output, diastolic and systolic pressure, respiration rate, and hormonal concentrations. Plausible VPs can provide prediction results and simulated data to begin to address the challenges for $\mathrm{HF}$ drug development. However, with observed clinical biomarker and response data, a set of plausible VPs can also be expanded and developed into a virtual patient population that quantitatively matches multiple summary statistics or distributions of the clinical observations [53-55]. These virtual populations can be used in silico trials to aid in a number of clinical development decisions with projections of anticipated population and trial variability. These insights including outcome distributions and quantitative population differences in the underlying mechanisms can be used for a more quantitative projection of trial outcomes when assessing doses, deciding how to stratify patients for therapy by their biomarkers, analyzing the contribution of components, and making decisions about combination 


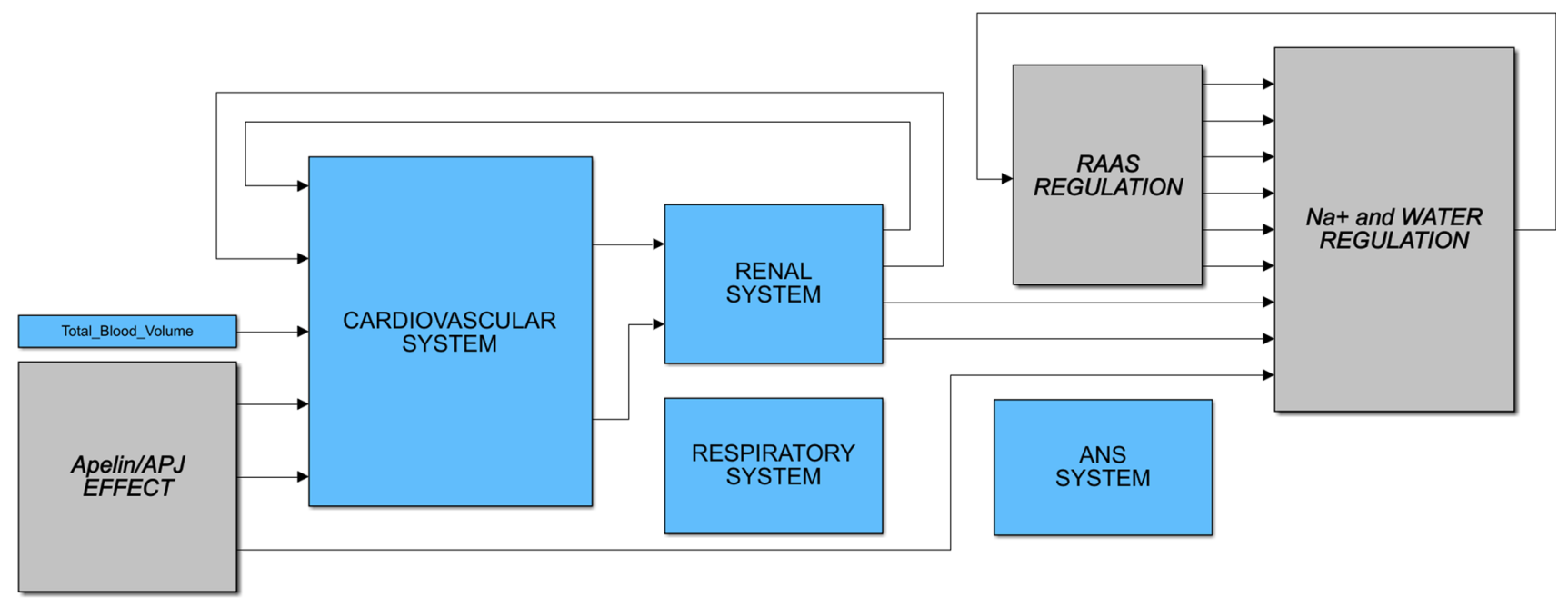

Fig. 1 Diagram of a quantitative systems pharmacology model of heart failure

therapy approaches, and deciding to advance or halt clinical development [56].

Another application of heart failure QSP modeling is predicting the progression of HF. Even if the HF patients are considered clinically stable when they are receiving treatment and show no physical signs and symptoms of worsening HF, HF treatments generally do not protect against progressive deterioration of cardiac function. The heart failure QSP model captures the progressive change and status of an HF patient with treatment or without treatment over time.

In addition to the QSP applications introduced, significant work has been done developing computational models of cardiac electrophysiology, cardiomyocytes and mechanics for guiding pharmaceutical therapy and deployment of devices, screening cardiac toxicity, and addressing heart failure questions [57]. It is also worth noting that HF can modulate organ blood flows that are important for tissue concentrations and clearance rates, and physiologically based pharmacokinetic (PBPK) models that model the effective clearance of HF drugs with the changes in organ perfusion associated with HF have been used to assess dosing as associated with the disease state $[58,59]$. Extension of PBPK models with simple pharmacodynamic (PD) models to create PBPK-PD models is one additional strategy to predict effects [60], and in addition modeling of cardiac tissue concentrations may further help to predict and assess cardiotoxicity [61]. While QSP approaches have also explicitly included distribution of a drug to target tissues, target binding, and mechanistic pharmacodynamic effects, in some cases it has also been useful to instead assess the impact of therapies through a direct in silico modulation of the target [50].

\section{Challenges and future directions for QSP modeling in heart failure}

$\mathrm{HF}$ is a widespread disease and poses a substantial medical need that is only partially being met. Overall, there are more than 26 million HF patients worldwide and more than half of them are 65 years or older. Half of HF patients die in 5 years, and a half of HF patients are re-hospitalized in 6 months. Currently, multiple assessments and tests are used for diagnosis, and half of the patients have no approved treatments. HF research is also expensive: a typical cardiovascular clinical trial can cost $\$ 72$ million, and half of phase III trials have failed. In addition, research in HF can take 30 years or even longer. As mentioned previously, heart failure often involves multiple organs and systems, results in many complications, and the relevant mechanisms for a patient may not be fully elucidated. Although there is already much clinical data related to HF, important readouts of clinical cardiac function and disease state such as ejection fraction, heart rate, and blood pressure may not be reported and generally are not available in a source electronic format where relationships can be more readily assessed. Clinical research in heart failure is challenging and investment in heart failure has declined [62].

Mechanistic cardiovascular computational modeling has been developed over several decades. For example, blood vessel hemodynamic models were published in 1959 [63], electrophysiological cardiac models were published in 1962 [64], electromechanical models of heart have been published since 1974 [65, 66], detailed computational biology of the heart from structure to function have been published since 1990s [67], and integrated cardiovascular models have been developed since 2000s [68, 69]. However, due to these challenges in HF, relatively few multiscale mechanistic models that incorporate the diverse 
pathophysiology from multiple length and time scales have been developed. For example, the QSP model of HF presented here captures multiple temporal scales, from modeling action potential at the time scale of milliseconds to modeling changes in cardiac out over years. For maximal mechanistic insight and ability to explore more therapies, biomarkers, and functions, HF models can in theory also utilize multiple physiological scales, from molecular interactions such as target engagement, signaling pathways [70], molecular cardiomyocyte processes including crossbridge cycling [51], cardiac tissue remodeling [50], up to systemic flow and blood pressure [50]. Current QSP modeling approaches only consider a limited set of components at the molecular through physiological scale. They can provide quantitative predictions as well as hypotheses for HF study, but the limit of resolution of the understanding they provide may be determined in part by the level of mathematical abstraction that mechanisms are incorporated at. Multiscale models of HF including additional processes incorporated explicitly at the molecular, cellular, tissue, and organ levels would therefore help to investigate additional contributing mechanisms and potential biomarkers in silico to deepen the mechanistic understanding of HF. Ultimately, a process of iterative model development, informed by assessment of model behaviors and available data, may help to further advance HF QSP.

\section{Machine learning in heart failure}

\section{Applications for machine learning in heart failure}

The goals of applying ML to medicine include improving the detection and classification of disease, making better predictions, and improving the personalization of medicine [15]. ML has been applied to HF to reduce cost by improving existing diagnostic and treatment support systems [71]. Current HF diagnosis and management rely upon patients' history, including their physical examination, and both laboratory and imaging data [72]. Learning from existing data, machine learning methods were applied to improve the accuracy and efficiency in predictions in $\mathrm{HF}$ diagnosis [73-75], readmission rate [76, 77], mortality rate [78, 79], and hospitalization rate [80]. While conventional statistical models have been used in heart failure, current state-of-art machine learning and deep learning techniques provide more powerful tools boosting the predictive accuracy [81]. A classification model was built by using a support vector machine (SVM) to classify all patients into three groups: the healthy group, the HF-prone group, and the HF group. Large amounts of physical examination records were used to build the ML model, including heart rate variability test, echocardiography test, electrocardiography test, chest radiography test, six minute walk test, and physical test [82]. Individualized treatment and healthy living choices can be suggested for high-risk patients predicted by ML from electronic health records [83]. Unsupervised clustering can identify phenotype groups in heart failure in baseline clinical characteristics, biomarker values, measures of left and right ventricular structure, and function, and the primary outcome occurrence $[46,84,85]$. The classification of phenotypically heterogeneous HF might aid in optimizing the rate of responders to specific therapies. Rather than the regression or classification tasks, machine learning models can also provide significant analysis on HF. For example, random forest methods can analyze the importance of each feature. The left ventricular ejection fraction was successfully identified as the most relevant feature in predicting the survival of patients [86]. Machine learning models were used to analyze heart failure patients, providing various outputs such as an HF severity evaluation, HF-type prediction, as well as a management interface that compares the different patients' follow-ups [87].

Many technologies can be used to assist the diagnosis of $\mathrm{HF}$, but there is also heterogeneity in clinical diagnoses due to differences between clinicians. Machine learning may help to improve the accuracy of diagnosis. LVEF is a measurement, expressed as a percentage, of how much blood the left ventricle pumps out with each contraction. Empirically, LVEF is an important classifier for HF [88]. However, patients with normal LVEF may have HF, termed HFpEF. The spatiotemporal variations of LV strain rate during rest and exercise can be used to identify patients with HFpEF and to provide an objective diagnostic classification. The analysis of such left ventricular long-axis function data via ML can improve the diagnosis and understanding of $\mathrm{HFpEF}$ [73, 74]. Electrocardiogram (ECG) is a non-invasive and simple diagnostic method that may demonstrate changes in congestive HF. However, the changes in ECG signal can also be difficult to detect accurately. Deep learning methods are therefore widely used to improve the accuracy in detecting congestive HF from ECG, where CNN [89] and LSTM [90] have been applied. Echocardiography is another standard tool for HF characterization and management, while it requires a skilled user and interpreter, and the interpretation of echocardiographic images has varying levels of subjectivity and inter-rater reliability $[15,91]$. Artificial intelligence (AI) technologies provide new possibilities for echocardiography to generate an accurate, consistent, and automated interpretation of echocardiograms, thus potentially reducing the risk of human error [92]. An ensemble machinelearning model, consisting of support vector machines, random forests, and artificial neural networks, was 
developed and a majority voting method was used for conclusive predictions based on echocardiographic images [93]. Combining echocardiography data and electronic health records, random forest achieved significantly higher prediction accuracy than logistic regression [94]. Wearable technologies recording cardiac function and machine learning algorithms can assess compensated and decompensated HF states by analyzing the cardiac response to submaximal exercise [95]. Heart sounds could be an economic and efficient method for a daily and home-based monitor for chronic HF. A model combining feature selection from a classic ML model and a downstream deep learning model was able to accurately identify new chronic HF patients through heart sounds [96].

\section{Challenges and future directions for machine learning in heart failure}

With increasingly available data, the accuracy of ML models can be improved in the data-driven approach. Data is an essential component in ML, QSP, and data-driven discovery. In general, a large amount of good quality training data can help to yield good accuracy of ML approaches. Deep learning has the ability for universal approximation to boost the predictive performance when large data sets are available. But challenges exist in machine/deep learning due to the data requirements. First, patient data and biomarkers collected from different sources or with different protocols may have strong variations and noise [15]. How to reduce the variations and align data from multiple sources together in one ML model is critical to the performance of the ML model. Data from different sources, such as different machines and hospitals, may present challenges [15]. Data from diverse and heterogenous sources typically involve different patient groups, inhomogeneous data collection techniques, and unspecified human errors, leading to nonuniform calibration, accuracy, and reliability, etc.

Because of their mechanistic nature, QSP models may help to flag and further check whether data from multiple sources are largely consistent or conflicting. Second, imbalanced data sets with low representation of a class of interest can significantly reduce the prediction accuracy and performance of both ML and QSP models. Tests that directly couple with the majority classes of the imbalanced data may be better predicted, but cases associated with the minority classes may not be accurately predicted. Many techniques on ML models can be applied to better work with imbalanced data [97]. For example, oversampling training data in the minority classes and using special loss function (e.g. focal loss [98]) are two popular approaches to enhance the predictive power of ML models on minority classes. Some analogous strategies may also facilitate QSP model calibration. For example in analogy to the focal loss strategy, statistical methods that ensure infrequent outcomes and biomarker observations associated with the infrequent outcomes are calibrated properly may be employed. Third, missing values in a dataset is a common phenomenon in potential data and biomarkers, especially for large datasets. Patients might skip or forget to take medicine or measurements. Most ML models require data that has the same dimension for individual components. Data imputation to fill out the missing values is necessary to the designs of an ML model to fully utilize all available information from data [99], and imputation strategies may also be useful for datasets for QSP model calibration. On the other hand, data may be limited number due to various reasons, for example if the data collection is expensive or the cases to be studied are rare. The complexity of the ML model needs to be appropriately selected to avoid overfitting. Similar considerations arise in QSP model calibration, and strategies to account for this have included both judicious selection of the number of parameters that are varied in a virtual population as well as model averaging [56].

Model interpretability is also extremely desirable for ML application on HF to reveal underlying mechanisms. However, the trade-off between model accuracy and model interpretability is a main challenge for ML approaches [100]. The integration of data-driven machine learning methods with mechanistic QSP modeling may be a component to overcome this obstacle.

\section{Machine learning-assisted QSP for heart failure}

In recent years, with the rise of ML and DL technology, QSP modelers have started to apply machine learning-assisted QSP for heart failure modeling (Fig. 2). One key area where we anticipate this will be important is relating clinical trial endpoints that are not mechanistic, such as survival, to endpoints that can be modeled explicitly. For example, we previously described the case where the left ventricular ejection fraction was found to be the most relevant feature in predicting the survival of patients [86]. In this case, the ML training and validation is done explicitly with the clinical data, but the insights can also be used to tie the QSP model outputs to additional clinical information of interest.

A second area where QSP and ML may be integrated is related to the calibration of model event rates using machine learning approaches, once a justifiable link between model outputs and clinical outcomes is set. In certain settings, we are given clinical event rate data reported from many trials with different (drug, dose) combinations, and some of these combinations may be 


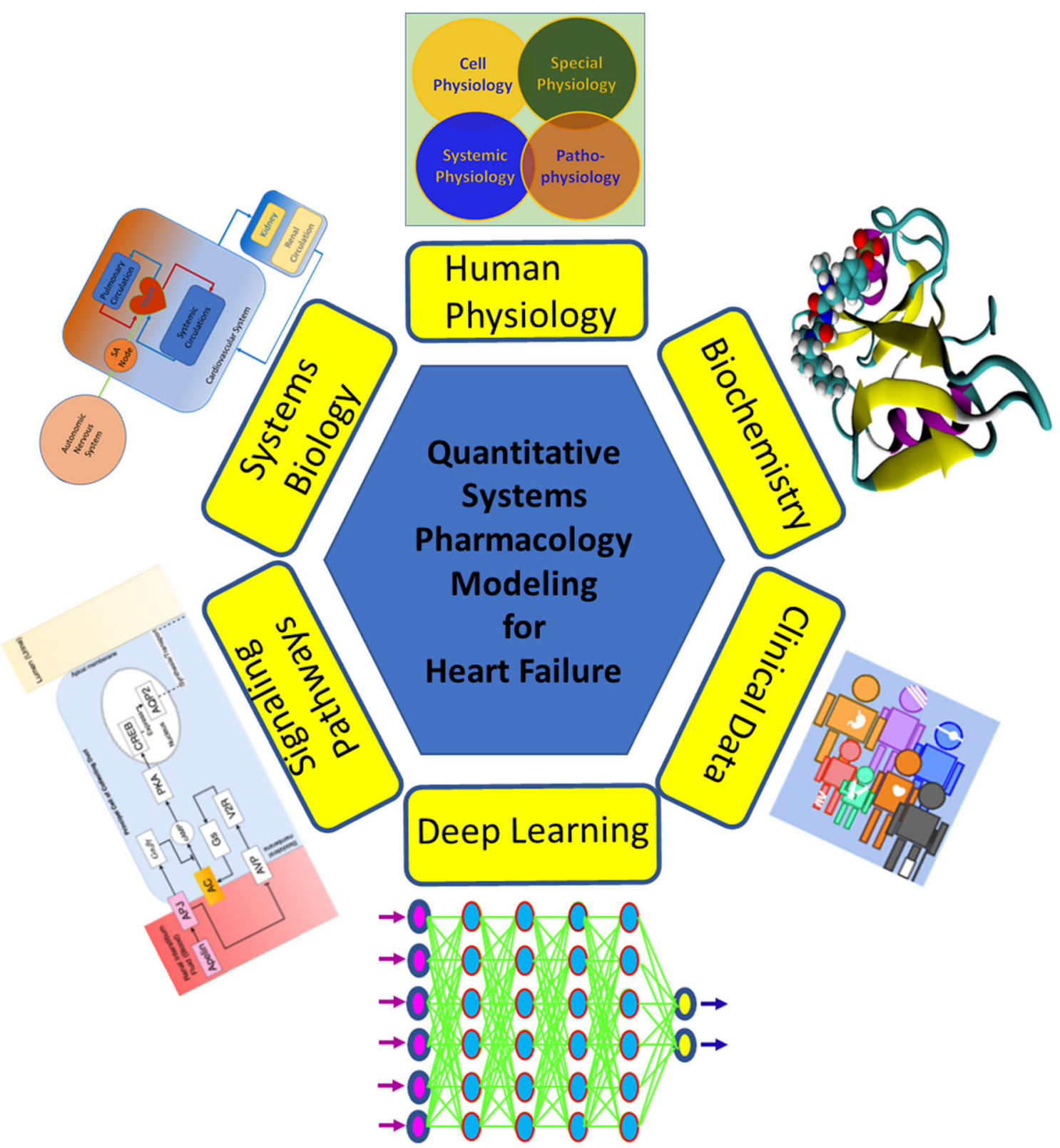

Fig. 2 An overview and illustration of machine learning assisted quantitative system pharmacology modeling for heart failure. It involves systems biology, physiology and pathophysiology, biochemistry, signaling pathways, and patient data

reported from multiple studies with varied outcomes. For each of these studies, we receive the event rate that was reported for the trial. This data can be used to tune an event prediction algorithm. With this strategy, machine learning models are trained at the population level of input data, generated from an ensemble of individual patient QSP simulations. Many ML algorithms can be developed for this purpose. For example, a logistic regression model that takes in the input field(s) for each virtual patient and outputs a probability of an event can be utilized. Other methods, including gradient, boosted decision tree
(GBDT), deep neural network (DNN), MDL, can be employed.

A third area where QSP and ML may be integrated is in the development of surrogate models of computationally challenging model subsystems. For example, if some subsystems operate on a relatively fast timescale that imposes smaller time steps on an ordinary differential equation solver, but analytically are not tractable to a good quasisteady-state approximation, a surrogate model developed with ML that recreates the input-output relationships of interest across all of the conditions to be explored may help to speed model simulations [101]. In our previous study, 
we used a simple Gaussian regression method as a surrogate for a signaling pathway saturation and desaturation mechanism before calibrating a QSP model of heart failure to publicly available clinical data for a competitor compound, then predicted the potential efficacy of our compound for decision making in the clinical development.

The application of mechanistic models often requires exploration of a high dimensional parameter space, and data-guided methods have been developed to both enable efficient characterization of this space and facilitate virtual population development [56, 102-105]. Highly efficient parameter exploration and model calibration strategies are a fourth area where ML strategies may further assist QSP. Recently, generative models, such as autoencoders, normalizing flows, and generative adversarial network [106], have been explored as a component of new strategies for statistical inference with mechanistic models, and also offer alternatives when the likelihood function is not analytically tractable [107]. Physics-informed neural networks (PINNs) can easily infer parameters and obtain accurate solution of the system with well-known governing Eqs. [108, 109]. PINNs have been applied to cardiac modeling [110]. Moreover, machine learning methods have been also applied to cardiac modeling for uncertainty quantification [111], model order reduction [112], surrogate generation and acceleration of large scale of simulations [113].

\section{Conclusions}

Although, to our knowledge, a QSP model that captures the full range of HF etiologies in good mechanistic detail that explicitly reproduces all proposed contributing factors across the molecular, cellular, tissue, and organ scales has not been developed, we can further develop current HF QSP models to work towards the goal of models capable of reproducing more clinical phenotypes, underlying causes, and calibration to more clinical interventions. We anticipate the utilization of ML approaches will help the advance towards this goal. ML will further bring together different approaches. QSP models that build in with new mechanisms and incorporate richer data will have a bright future.

Acknowledgements This work was supported in part by NIH grant GM126189, NSF grants DMS-2052983, DMS-1761320, and IIS1900473 , NASA grant 80NSSC21M0023, Michigan State Foundation, Bristol-Myers Squibb 65109.

Open Access This article is licensed under a Creative Commons Attribution 4.0 International License, which permits use, sharing, adaptation, distribution and reproduction in any medium or format, as long as you give appropriate credit to the original author(s) and the source, provide a link to the Creative Commons licence, and indicate if changes were made. The images or other third party material in this article are included in the article's Creative Commons licence, unless indicated otherwise in a credit line to the material. If material is not included in the article's Creative Commons licence and your intended use is not permitted by statutory regulation or exceeds the permitted use, you will need to obtain permission directly from the copyright holder. To view a copy of this licence, visit http://creativecommons. org/licenses/by/4.0/.

\section{References}

1. Denolin H, Kuhn H, Krayenbuehl H, Loogen F, Reale A (1983) The defintion of heart failure. Eur Heart J 4(7):445-448

2. Bhuiyan T, Maurer MS (2011) Heart failure with preserved ejection fraction: persistent diagnosis, therapeutic enigma. Curr Cardiovasc Risk Rep 5(5):440

3. Azevedo PS, Polegato BF, Minicucci MF, Paiva SA, Zornoff LA (2015) Cardiac remodeling: concepts, clinical impact, pathophysiological mechanisms and pharmacologic treatment. Arq Bras Cardiol 106:62-69

4. Tanai E, Frantz S (2011) Pathophysiology of heart failure. Compr Physiol 6(1):187-214

5. Anversa P, Kajstura J, Olivetti G (1996) Myocyte death in heart failure. Curr Opin Cardiol 11(3):245-251

6. Miller WL (2016) Fluid volume overload and congestion in heart failure: time to reconsider pathophysiology and how volume is assessed. Circulation 9(8):e002922

7. Mann DL (1999) Mechanisms and models in heart failure: a combinatorial approach. Circulation 100(9):999-1008

8. Leil TA, Ermakov S (2015) The emerging discipline of quantitative systems pharmacology. Front Pharmacol 6:129

9. Sorger PK, Allerheiligen SR, Abernethy DR, Altman RB, Brouwer KL, Califano A et al (2011) Quantitative and systems pharmacology in the post-genomic era: new approaches to discovering drugs and understanding therapeutic mechanisms. An $\mathrm{NIH}$ white paper by the QSP workshop group. NIH Bethesda, Bethesda

10. Bai JP, Schmidt BJ, Gadkar KG, Damian V, Earp JC, Friedrich $C$ et al (2021) FDA-Industry Scientific Exchange on assessing quantitative systems pharmacology models in clinical drug development: a meeting report, summary of challenges/gaps, and future perspective. Springer, Cham

11. Musuamba FT, Bursi R, Manolis E, Karlsson K, Kulesza A, Courcelles E et al (2020) Verifying and validating quantitative systems pharmacology and in silico models in drug development: current needs, gaps, and challenges. CPT 9(4):195

12. Jordan MI, Mitchell TM (2015) Machine learning: Trends, perspectives, and prospects. Science 349(6245):255-260

13. Wei G-W (2019) Protein structure prediction beyond AlphaFold. Nat Mach Intell 1(8):336-337

14. Carbonell JG, Michalski RS, Mitchell TM (1983) An overview of machine learning. Mach Learn 1983:3-23

15. Olsen CR, Mentz RJ, Anstrom KJ, Page D, Patel PA (2020) Clinical applications of machine learning in the diagnosis, classification, and prediction of heart failure. Am Heart $\mathbf{J}$ 229:1-17

16. Strahler AN (1957) Quantitative analysis of watershed geomorphology. Eos Trans Am Geophys Union 38(6):913-920

17. Kleinbaum DG, Dietz K, Gail M, Klein M, Klein M (2002) Logistic regression. Springer, Cham

18. Cortes C, Vapnik V (1995) Support-vector networks. Mach Learn 20(3):273-297 
19. Safavian SR, Landgrebe D (1991) A survey of decision tree classifier methodology. IEEE Trans Syst Man cybern 21(3):660-674

20. Ho TK, editor Random decision forests. In: Proceedings of 3rd international conference on document analysis and recognition; 1995: IEEE

21. Chen T, He T, Benesty M, Khotilovich V, Tang Y, Cho H. Xgboost: extreme gradient boosting. $\mathrm{R}$ package version 04-2. 2015;1(4)

22. Schmidhuber J (2015) Deep learning in neural networks: an overview. Neural Netw 61:85-117

23. LeCun Y, Bengio Y (1995) Convolutional networks for images, speech, and time series. The Handb Brain Theory Neural Netw 3361(10): 1995

24. Gers FA, Schmidhuber J, Cummins F (2000) Learning to forget: continual prediction with LSTM. Neural Comput 12(10):2451-2471

25. Chung J, Gulcehre C, Cho K, Bengio Y. Empirical evaluation of gated recurrent neural networks on sequence modeling. arXiv preprint arXiv: 14123555.2014

26. Tetko IV, Karpov P, Van Deursen R, Godin G (2020) State-ofthe-art augmented NLP transformer models for direct and single-step retrosynthesis. Nature Commun 11(1):1-11

27. Van Der Maaten L, Postma E, Van den Herik J (2009) Dimensionality reduction: a comparative. J Mach Learn Res 10(66-71): 13

28. Wold S, Esbensen K, Geladi P (1987) Principal component analysis. Chemom Intell Lab Syst 2(1-3):37-52

29. Lee DD, Seung HS (1999) Learning the parts of objects by nonnegative matrix factorization. Nature 401(6755):788-791

30. Van der Maaten L, Hinton G (2008) Visualizing data using t-SNE. J Mach Learn Res 9(11):2579

31. Vincent P, Larochelle H, Lajoie I, Bengio Y, Manzagol P-A, Bottou L (2010) Stacked denoising autoencoders: Learning useful representations in a deep network with a local denoising criterion. J Mach Learn Res 11(12):3371

32. McInnes L, Healy J, Melville J. Umap: Uniform manifold approximation and projection for dimension reduction. arXiv preprint arXiv:180203426. 2018

33. Hozumi Y, Wang R, Yin C, Wei G-W (2021) UMAP-assisted $\mathrm{K}$-means clustering of large-scale SARS-CoV-2 mutation datasets. Comput Biol Med 131:104264

34. Hamerly G, Elkan C (2004) Learning the k in k-means. Adv Neural Inf Process Syst 16:281-288

35. Zhang Q, Couloigner I (2005) A new and efficient k-medoid algorithm for spatial clustering. International conference on computational science and its applications. Springer, Berlin

36. Murtagh F, Contreras P (2012) Algorithms for hierarchical clustering: an overview. Wiley Interdiscip Rev 2(1):86-97

37. Traag VA, Waltman L, Van Eck NJ (2019) From Louvain to Leiden: guaranteeing well-connected communities. Sci Rep 9(1):1-12

38. Blondel VD, Guillaume J-L, Lambiotte R, Lefebvre E (2008) Fast unfolding of communities in large networks. J Stat Mech 2008(10):P10008

39. Schubert E, Sander J, Ester M, Kriegel HP, Xu X (2017) DBSCAN revisited, revisited: why and how you should (still) use DBSCAN. ACM Trans Database Syst 42(3):1-21

40. Sha Y, Wang S, Zhou P, Nie Q (2020) Inference and multiscale model of epithelial-to-mesenchymal transition via single-cell transcriptomic data. Nucleic Acids Res 48(17):9505-9520

41. Kiselev VY, Kirschner K, Schaub MT, Andrews T, Yiu A, Chandra $\mathrm{T}$ et al (2017) SC3: consensus clustering of single-cell RNA-seq data. Nat Methods 14(5):483-486

42. Kuang D, Ding C, Park H, editors. Symmetric nonnegative matrix factorization for graph clustering. In: Proceedings of the
2012 SIAM international conference on data mining; 2012: SIAM

43. Oller-Moreno S, Kloiber K, Machart P, Bonn S. Algorithmic advances in machine learning for single cell expression analysis. Current Opinion in Systems Biology. 2021

44. Saxena A, Prasad M, Gupta A, Bharill N, Patel OP, Tiwari A et al (2017) A review of clustering techniques and developments. Neurocomputing 267:664-681

45. Zhong Y, Ma A, soon Ong Y, Zhu Z, Zhang L (2018) Computational intelligence in optical remote sensing image processing. Appl Soft Comput 64:75-93

46. Cikes M, Sanchez-Martinez S, Claggett B, Duchateau N, Piella G, Butakoff C et al (2019) Machine learning-based phenogrouping in heart failure to identify responders to cardiac resynchronization therapy. Eur J Heart Fail 21(1):74-85

47. Schmidt BJ, Papin JA, Musante CJ (2013) Mechanistic systems modeling to guide drug discovery and development. Drug Discov Today 18(3-4):116-127

48. Powell LM, Lo A, Cole MS, Trimmer J (2007) Application of predictive biosimulation to the study of atherosclerosis: development of the cardiovascular PhysioLab platform and evaluation of CETP inhibitor therapy. Proc FOSBE 2007:9-12

49. Wahba K, Lo A, Kadambi A, Powell LM (2011) Clinical trial simulations of dyslipidemic patients in a mechanistic model of cardiovascular disease predict little impact on CHD events by CETP inhibitors. Am Heart Assoc 2011:A9560

50. Yu H, Basu S, Hallow KM (2020) Cardiac and renal function interactions in heart failure with reduced ejection fraction: A mathematical modeling analysis. PLoS Comput Biol 16(8):e1008074

51. Tewari SG, Bugenhagen SM, Vinnakota KC, Rice JJ, Janssen PM, Beard DA (2016) Influence of metabolic dysfunction on cardiac mechanics in decompensated hypertrophy and heart failure. J Mol Cell Cardiol 94:162-175

52. Pocock SJ, Stone GW (2016) The primary outcome fails-what next? N Engl J Med 375(9):861-870

53. Woodhead JL, Howell BA, Yang Y, Harrill AH, Clewell HJ, Andersen ME et al (2012) An analysis of N-acetylcysteine treatment for acetaminophen overdose using a systems model of drug-induced liver injury. $J$ Pharmacol Exp Ther 342(2):529-540

54. Allen R, Rieger TR, Musante CJ (2016) Efficient generation and selection of virtual populations in quantitative systems pharmacology models. CPT 5(3):140-146

55. Cheng Y, Thalhauser CJ, Smithline S, Pagidala J, Miladinov M, Vezina HE et al (2017) QSP toolbox: computational implementation of integrated workflow components for deploying multi-scale mechanistic models. AAPS J 19(4):1002-1016

56. Cheng Y, Straube R, Alnaif EA, Huang L, Leil AT, Schmidt JB (2021) Virtual populations for quantitative systems pharmacology models. Systems Medicine, Springer

57. Niederer SA, Lumens J, Trayanova NA (2019) Computational models in cardiology. Nat Rev Cardiol 16(2):100-111

58. Rasool MF, Khalil F, Läer S (2015) A physiologically based pharmacokinetic drug-disease model to predict carvedilol exposure in adult and paediatric heart failure patients by incorporating pathophysiological changes in hepatic and renal blood flows. Clin Pharm 54(9):943-962

59. Mangoni AA, Jarmuzewska EA (2019) The influence of heart failure on the pharmacokinetics of cardiovascular and non-cardiovascular drugs: a critical appraisal of the evidence. Br J Clin Pharmacol 85(1):20-36

60. Lang J, Vincent L, Chenel M, Ogungbenro K, Galetin A (2020) Simultaneous ivabradine parent-metabolite PBPK/PD modelling using a Bayesian estimation method. AAPS J 22(6):1-16 
61. Tylutki Z, Mendyk A, Polak S (2018) Mechanistic physiologically based pharmacokinetic (PBPK) model of the heart accounting for inter-individual variability: development and performance verification. J Pharm Sci 107(4):1167-1177

62. Packer M (2016) Unbelievable folly of clinical trials in heart failure: the inconvenient truth about how investigators and guidelines weigh evidence. Circulation 9(4):e002837

63. Grodins FS (1959) Integrative cardiovascular physiology: a mathematical synthesis of cardiac and blood vessel hemodynamics. Q Rev Biol 34(2):93-116

64. Noble D (1962) A modification of the Hodgkin-Huxley equations applicable to Purkinje fibre action and pacemaker potentials. J Physiol 160(2):317-352

65. Kaufmann R, Bayer R, Fürniss T, Krause H, Tritthart H (1974) Calcium-movement controlling cardiac contractility. II. Analog computation of cardiac excitation-contraction coupling on the basis of calcium kinetics in a multi-compartment model. J Mol Cell Cardiol 6(6):543-559

66. Trayanova NA, Rice JJ (2011) Cardiac electromechanical models: from cell to organ. Front Physiol 2:43

67. McCulloch A, Bassingthwaighte J, Hunter P, Noble D (1998) Computational biology of the heart: from structure to function. Prog Biophys Mol Biol 69:153

68. Ursino M, Magosso E (2003) Short-term autonomic control of cardiovascular function: a mini-review with the help of mathematical models. J Integr Neurosci 2(02):219-247

69. Cheng L, Ivanova O, Fan H-H, Khoo MC (2010) An integrative model of respiratory and cardiovascular control in sleep-disordered breathing. Respir Physiol Neurobiol 174(1-2):4-28

70. Khalilimeybodi A, Daneshmehr A, Sharif-Kashani B (2018) Investigating $\beta$-adrenergic-induced cardiac hypertrophy through computational approach: classical and non-classical pathways. J Physiol Sci 68(4):503-520

71. Awan SE, Sohel F, Sanfilippo FM, Bennamoun M, Dwivedi G (2018) Machine learning in heart failure: ready for prime time. Curr Opin Cardiol 33(2):190-195

72. UK NA-A, Atherton JJ, Bauersachs J, UK AJC, Carerj S, Ceconi C et al (2016) 2016 ESC Guidelines for the diagnosis and treatment of acute and chronic heart failure. Eur Heart $\mathrm{J}$ 37:2129-2200

73. Tabassian M, Sunderji I, Erdei T, Sanchez-Martinez S, Degiovanni A, Marino P et al (2018) Diagnosis of heart failure with preserved ejection fraction: machine learning of spatiotemporal variations in left ventricular deformation. J Am Soc Echocardiogr 31(12): 1272-1284 (e9)

74. Sanchez-Martinez S, Duchateau N, Erdei T, Kunszt G, Aakhus S, Degiovanni A et al (2018) Machine learning analysis of left ventricular function to characterize heart failure with preserved ejection fraction. Circulation 11(4):e007138

75. Alotaibi FS (2019) Implementation of machine learning model to predict heart failure disease. Int J Adv Comput Sci Appl 10(6):261-268

76. Mortazavi BJ, Downing NS, Bucholz EM, Dharmarajan K, Manhapra A, Li S-X et al (2016) Analysis of machine learning techniques for heart failure readmissions. Circulation 9(6):629-640

77. Frizzell JD, Liang L, Schulte PJ, Yancy CW, Heidenreich PA, Hernandez AF et al (2017) Prediction of 30-day all-cause readmissions in patients hospitalized for heart failure: comparison of machine learning and other statistical approaches. JAMA Cardiol 2(2):204-209

78. Jing L, Ulloa Cerna AE, Good CW, Sauers NM, Schneider G, Hartzel DN et al (2020) A machine learning approach to management of heart failure populations. Heart Fail 8(7):578-587
79. Adler ED, Voors AA, Klein L, Macheret F, Braun OO, Urey MA et al (2020) Improving risk prediction in heart failure using machine learning. Eur J Heart Fail 22(1):139-147

80. Angraal S, Mortazavi BJ, Gupta A, Khera R, Ahmad T, Desai NR et al (2020) Machine learning prediction of mortality and hospitalization in heart failure with preserved ejection fraction. JACC 8(1):12-21

81. Shin S, Austin PC, Ross HJ, Abdel-Qadir H, Freitas C, Tomlinson $\mathrm{G}$ et al (2021) Machine learning vs. conventional statistical models for predicting heart failure readmission and mortality. ESC Heart Fail 8(1):106-115

82. Yang G, Ren Y, Pan Q, Ning G, Gong S, Cai G et al, editors. A heart failure diagnosis model based on support vector machine (2010) 3rd International Conference on Biomedical Engineering and Informatics; 2010: IEEE

83. Panahiazar M, Taslimitehrani V, Pereira N, Pathak J (2015) Using EHRs and machine learning for heart failure survival analysis. Stud Health Technol Inform 216:40

84. Segar MW, Patel KV, Ayers C, Basit M, Tang WW, Willett D et al (2020) Phenomapping of patients with heart failure with preserved ejection fraction using machine learning-based unsupervised cluster analysis. Eur J Heart Fail 22(1):148-158

85. Hedman ÅK, Hage C, Sharma A, Brosnan MJ, Buckbinder L, Gan L-M et al (2020) Identification of novel pheno-groups in heart failure with preserved ejection fraction using machine learning. Heart 106(5):342-349

86. Chicco D, Jurman G (2020) Machine learning can predict survival of patients with heart failure from serum creatinine and ejection fraction alone. BMC Med Inf Decis Mak 20(1):16

87. Guidi G, Pettenati MC, Melillo P, Iadanza E (2014) A machine learning system to improve heart failure patient assistance. IEEE J Biomed Health Inf 18(6):1750-1756

88. Yancy CW, Jessup M, Bozkurt B, Butler J, Casey DE, Drazner MH et al (2013) 2013 ACCF/AHA guideline for the management of heart failure: a report of the American College of Cardiology Foundation/American Heart Association Task Force on Practice Guidelines. J Am Coll Cardiol 62(16):e147-e239

89. Acharya UR, Fujita H, Oh SL, Hagiwara Y, Tan JH, Adam M et al (2019) Deep convolutional neural network for the automated diagnosis of congestive heart failure using ECG signals. Appl Intell 49(1):16-27

90. Wang L, Zhou X (2019) Detection of congestive heart failure based on LSTM-based deep network via short-term RR intervals. Sensors 19(7):1502

91. Selmeryd J, Henriksen E, Leppert J, Hedberg P (2016) Interstudy heterogeneity of definitions of diastolic dysfunction severely affects reported prevalence. Eur Heart J 17(8):892-899

92. Alsharqi M, Woodward W, Mumith J, Markham D, Upton R, Leeson P (2018) Artificial intelligence and echocardiography. Echo Res Pract 5(4):R115-R125

93. Narula S, Shameer K, Salem Omar AM, Dudley JT, Sengupta PP (2016) Machine-learning algorithms to automate morphological and functional assessments in 2D echocardiography. J Am Coll Cardiol 68(21):2287-2295

94. Samad MD, Ulloa A, Wehner GJ, Jing L, Hartzel D, Good CW et al (2019) Predicting survival from large echocardiography and electronic health record datasets: optimization with machine learning. JACC 12(4):681-689

95. Inan OT, Baran Pouyan M, Javaid AQ, Dowling S, Etemadi M, Dorier A et al (2018) Novel wearable seismocardiography and machine learning algorithms can assess clinical status of heart failure patients. Circulation 11(1):e004313

96. Gjoreski M, Gradišek A, Budna B, Gams M, Poglajen G (2020) Machine learning and end-to-end deep learning for the detection of chronic heart failure from heart sounds. IEEE Access 8:20313-20324 
97. Kaur H, Pannu HS, Malhi AK (2019) A systematic review on imbalanced data challenges in machine learning: Applications and solutions. ACM Comput Surv 52(4):1-36

98. Lin T-Y, Goyal P, Girshick R, He K, Dollár P, editors. Focal loss for dense object detection. Proceedings of the IEEE international conference on computer vision; 2017

99. Efron B (1994) Missing data, imputation, and the bootstrap. J Am Stat Assoc 89(426):463-475

100. Johansson U, Sönströd C, Norinder U, Boström H (2011) Tradeoff between accuracy and interpretability for predictive in silico modeling. Future Med Chem 3(6):647-663

101. Hasenauer J, Jagiella N, Hross S, Theis FJ (2015) Data-driven modelling of biological multi-scale processes. J Coupled Syst Multiscale Dyn 3(2):101-121

102. Zamora-Sillero E, Hafner M, Ibig A, Stelling J, Wagner A (2011) Efficient characterization of high-dimensional parameter spaces for systems biology. BMC Syst Biol 5(1):1-22

103. Dixit PD, Lyashenko E, Niepel M, Vitkup D (2020) Maximum entropy framework for predictive inference of cell population heterogeneity and responses in signaling networks. Cell Syst 10(2):204-12.e8

104. Catanach TA, Beck JL (2018) Bayesian updating and uncertainty quantification using sequential tempered $\mathrm{mcmc}$ with the rank-one modified metropolis algorithm. arXiv preprint arXiv: 180408738.

105. Sivia DS, Skilling J (2006) Nested sampling. In: Data analysis: a Bayesian tutorial, 2nd edn. Oxford University Press, Oxford, New York, pp 181-208
106. Parikh J, Kozloski J, Gurev V. Integration of AI and mechanistic modeling in generative adversarial networks for stochastic inverse problems. arXiv preprint arXiv:200908267.2020

107. Cranmer K, Brehmer J, Louppe G (2020) The frontier of simulation-based inference. Proc Natl Acad Sci 117(48): 30055-30062

108. Raissi M, Perdikaris P, Karniadakis GE (2019) Physics-informed neural networks: a deep learning framework for solving forward and inverse problems involving nonlinear partial differential equations. J Comput Phys 378:686-707

109. Yazdani A, Lu L, Raissi M, Karniadakis GE (2020) Systems biology informed deep learning for inferring parameters and hidden dynamics. PLoS Comput Biol 16(11):e1007575

110. Sahli Costabal F, Yang Y, Perdikaris P, Hurtado DE, Kuhl E (2020) Physics-informed neural networks for cardiac activation mapping. Front Phys 8:42

111. Costabal FS, Choy J, Sack KL, Guccione JM, Kassab G, Kuhl E (2019) Multiscale characterization of heart failure. Acta Biomater 86:66-76

112. Di Achille P, Parikh J, Khamzin S, Solovyova O, Kozloski J, Gurev V (2020) Model order reduction for left ventricular mechanics via congruency training. Plos one 15(1):e0219876

113. Costabal FS, Perdikaris P, Kuhl E, Hurtado DE (2019) Multifidelity classification using Gaussian processes: accelerating the prediction of large-scale computational models. Comput Methods Appl Mech Eng 357:112602

Publisher's Note Springer Nature remains neutral with regard to jurisdictional claims in published maps and institutional affiliations. 